\title{
Plano de gestão integrada de resíduos sólidos de São Paulo na perspectiva da avaliação ambiental estratégica
}

\author{
São Paulo integrated management plan of solid waste in the perspective of \\ the strategic environmental assessment
}

Maria Cristina Mendonça Vieira[a], Amarilis Lucia Casteli Figueiredo Gallardo[b,c] (B), Alexandre de Oliveira e Aguiarlc] [i], Guilherme Leite Gaudereto[d] [1]

[a] Universidade de São Paulo (USP), Escola de Artes, Ciências e Humanidades, São Paulo, SP, Brasil

[b] Universidade de São Paulo (USP), Escola Politécnica, Departamento de Engenharia Hidráulica e Ambiental, São Paulo, SP, Brasil

[c] Universidade Nove de Julho (UNINOVE), São Paulo, SP, Brasil

[d] Universidade de São Paulo (USP), Escola Politécnica, Programa de Pós-graduação em Engenharia Civil, São

Paulo, SP, Brasil

Como citar: Vieira, M. C. M., Gallardo, A. L. C. F., Aguiar, A. O., \& Gaudereto, G. L. (2019). Plano de gestão integrada de resíduos sólidos de São Paulo na perspectiva da avaliação ambiental estratégica. urbe. Revista Brasileira de Gestão Urbana, 11, e20180155. https://doi.org/10.1590/2175-3369.011.e20180155

\section{Resumo}

Planos de Gestão Integrada de Resíduos Sólidos são instrumentos inovadores de planejamento e gestão de resíduos urbanos, representando um avanço importante, promovido pela Política Nacional de Resíduos Sólidos, para a sustentabilidade. A Avaliação Ambiental Estratégica é um instrumento que visa integrar a variável socioambiental em políticas, planos e programas. A partir da questão "Como a Avaliação Ambiental Estratégica pode contribuir para o processo de elaboração de planos municipais de gestão de resíduos sólidos no contexto da Política Nacional de Resíduos Sólidos?", pretende-se analisar: a adequação do plano de São Paulo às orientações internacionais de boas práticas de Avaliação Ambiental Estratégica; e como os objetivos estratégicos da política nacional influenciam o plano local, demonstrando evidências de tiering no planejamento do setor. Tiering é o encadeamento da temática ambiental nos diferentes níveis do planejamento. A maioria dos elementos-chave de boas práticas de Avaliação Ambiental Estratégica e as evidências de tiering na cadeia decisória do planejamento da política para o plano municipal são observadas no Plano de Gestão Integrada de Resíduos Sólidos. Aspectos relevantes da Avaliação Ambiental Estratégica não constatados e a perspectiva dos benefícios associados permitem recomendar que o instrumento seja sistematicamente empregado no planejamento e na gestão de resíduos da escala nacional para a local.

Palavras-chave: Avaliação ambiental estratégica. Gestão de resíduos sólidos. Plano de gestão integrada de resíduos sólidos. Política Nacional de Resíduos Sólidos.

MCMV é graduada em Gestão Ambiental, e-mail: crissmendonca@gmail.com

ALCFG é pós-doutora em Ciências Ambientais, doutora e mestre em Engenharia, e-mail: amarilislcfgallardo@gmail.com AOA é doutor e mestre em Saúde Pública, e-mail: alexandre@aguiar.eng.br

GLG é doutorando em Engenharia, mestre em Gestão Ambiental e Sustentabilidade, e-mail: guilherme.gaudereto@usp.br 


\section{Abstract}

Integrated Management Plans of Solid Waste are an innovative instrument management for urban waste, representing an important advance for sustainability guided by the National Solid Waste Policy. Strategic Environmental Assessment is an instrument for integrating the socio-environmental issues into policies, plans, and programs. From this question - How can Strategic Environmental Assessment contribute to the process of preparing municipal solid waste management plans in the context of the National Solid Waste Policy? - we analyzed: the suitability of the São Paulo waste plan to the international guidelines for Strategic Environmental Assessment good practices; and how the strategic objectives of the national policy influence the local plan by showing evidence of tiering in this planning sector. Tiering is understood as the chaining of environmental issues at different levels of planning. Some key-elements of Strategic Environmental Assessment good practices and the evidence of tiering from policy to municipal plan are observed in the São Paulo waste plan. Most relevant themes of Strategic Environmental Assessment are observed. Its potential benefits can recommend that Strategic Environmental Assessment has to be systematically used in the planning and management of solid waste from the national to the local scale.

Keywords: Strategic environmental assessment. Solid waste management. Integrated solid waste management plan. National Solid Waste Policy.

\section{Introdução}

O planejamento da gestão de resíduos sólidos urbanos é um dos desafios enfrentados pelos gestores públicos municipais (Jacobi \& Besen, 2011), devendo integrar desde a não geração até a disposição final, em ativa cooperação entre governo, iniciativa privada e sociedade (Zanta \& Ferreira, 2003). Para Silva et al. (2017), a gestão de resíduos sólidos urbanos é fator determinante para o desenvolvimento dos municípios. A complexidade da gestão de resíduos sólidos quanto aos impactos ambientais e às repercussões sociais, governamentais, territoriais e técnicas encontra respaldo na inovadora Política Nacional de Resíduos Sólidos (PNRS) (Marchi, 2015).

A PNRS (Lei no c 12.305/2010) estabelece as diretrizes mínimas para o equacionamento da gestão de resíduos sólidos municipais (Campos, 2012). Entre seus instrumentos mais relevantes, encontra-se o Plano de Gestão Integrada de Resíduos Sólidos (PGIRS), documento técnico obrigatório para os municípios. Para atender a essa política, São Paulo apresentou, em 2014, seu PGIRS (São Paulo, 2014). Contudo, Maiello et al. (2018) ressaltam as dificuldades para integração entre entes federativos na implementação de políticas públicas desde as diretrizes formuladas em nível nacional até as instâncias executoras em nível local, tendo como base a PNRS.

No campo do planejamento, a Avaliação Ambiental Estratégica (AAE) proporciona a discussão das alternativas estratégicas para a inserção de aspectos ambientais e de sustentabilidade no planejamento e seu desenvolvimento (Partidário, 2012). Promulgada na Europa em 2004, é utilizada em mais de 60 países (Tetlow \& Hanusch, 2012). No Brasil, a AAE é não mandatória e caracteriza-se por poucas experiências de aplicação, sendo considerada em evolução por Montaño et al. (2014).

De acordo com Malvestio et al. (2012), a AAE tem oportunidade de cumprir relevante papel no planejamento e na gestão de resíduos pelo aumento da demanda para elaboração de planos de resíduos sólidos em função da promulgação da PNRS. Estudos dessa natureza vêm sendo realizados em locais em que a prática de AAE está consolidada. Desmond (2009), por sua vez, utilizou a gestão de resíduos da Irlanda para avaliar o alcance da AAE na definição de alternativas estratégicas quanto aos desdobramentos desse planejamento setorial em seus vários níveis hierárquicos. Enquanto Fischer et al. (2011) discutiram a aplicação da AAE às estratégias municipais de gestão de resíduos na Inglaterra, identificando oportunidades para a valorização da hierarquia de gestão de resíduos. Para Desmond (2009) e Fischer et al. (2011), os benefícios da AAE são alcançados quando sua aplicação é sistemática e contínua dentro de um sistema de planejamento escalonado. 
Na ausência de marco regulatório da AAE no país, Sánchez (2017) destaca o uso da AAE para articulação vertical entre os níveis do planejamento, considerando impactos socioambientais, formulando opções estratégicas e contribuindo para o desenvolvimento sustentável. Vários estudos brasileiros têm contribuído com reflexões para avançar na consolidação da prática de AAE, como os desenvolvidos por Sánchez \& Silva-Sánchez (2008), Montaño et al. (2014) e Nadruz et al. (2018), enquanto outros têm buscado compreender o alcance e os benefícios da AAE ao planejamento regional e setorial, como Malvestio et al. (2012), Gallardo et al. (2016) e Rizzo et al. (2017).

Esta pesquisa busca contribuir com essa última corrente de trabalhos, selecionando o planejamento de resíduos como objeto de análise na perspectiva de discussão do tiering (hierarquização ou encadeamento, em tradução livre) no planejamento setorial, em continuidade às pesquisas de Sánchez \& Silva-Sánchez (2008) e Turco \& Gallardo (2018).

De acordo com as boas práticas internacionais (Partidário, 2012), a aplicação da AAE deve ser realizada concomitantemente ao desenvolvimento do planejamento por equipe multidisciplinar e sujeita à ampla e aberta participação pública (Tetlow \& Hanusch, 2012). Como o PGIRS de São Paulo não foi deliberadamente elaborado segundo essas premissas, buscou-se identificar se a perspectiva da AAE e os potenciais benefícios associados encontram-se incluídos nesse plano. Estabeleceu-se como questão de pesquisa: como a AAE pode contribuir para o processo de elaboração de planos municipais de gestão de resíduos sólidos no contexto da PNRS? Foram estabelecidos dois objetivos: analisar a adequação do PGIRS de São Paulo às orientações internacionais de boas práticas de AAE; e analisar como os objetivos estratégicos da PNRS influenciam o PGIRS, demonstrando evidências de tiering na cadeia de decisões no planejamento nacional de resíduos sólidos.

\section{Gestão de resíduos sólidos no Brasil}

A produção e o descarte de resíduos colocam em risco a sustentabilidade. A geração mundial de resíduos sólidos municipais está em torno de 1,32 bilhão de toneladas por ano e, até 2025 , a previsão é de 2,2 bilhões de toneladas (Hoornweg \& Bhada, 2012).

A quantidade de resíduos sólidos urbanos gerada no país, em 2016, foi de 78,3 milhões de toneladas, das quais 29,7 milhões de toneladas foram despejadas em lixões e aterros controlados. Além disso, quase $42 \%$ dos resíduos coletados não são destinados a aterros sanitários, empregando soluções inadequadas de disposição final (ABRELPE, 2017).

Esse cenário associa impactos ambientais, como poluição do ar, contaminação do solo, das águas superficiais e de lençóis freáticos, riscos à saúde pública, problemas socioeconômicos, poluição visual, mau odor e desvalorização imobiliária (Espinosa \& Silvas, 2014), que se estendem para além das áreas de disposição final, afetando a população em geral.

A Organização das Nações Unidas (ONU) vem preconizando que a geração de resíduos precisa ser minimizada (ONU, 1992; UNEP, 2011). Essa diretriz desdobra-se em metas de políticas públicas na União Europeia, no Reino Unido (Bulkeley \& Gregson, 2009) e no Brasil (2010). Essa abordagem implica mudanças nos padrões de produção e consumo (ONU, 1992; Philippi \& Aguiar, 2018), incluindo a demanda por mais produtos reciclados (Bulkeley \& Gregson, 2009; UNEP, 2011), ou seja, uma cultura mais voltada ao compartilhamento em detrimento da posse e a incentivos de mercado, de tecnologia e de conscientização (UNEP, 2011). Uma interação efetiva entre a administração pública e a privada requer soluções mais complexas. Nesse contexto, insere-se a AAE, que vem contribuindo para que a elaboração de políticas públicas e seus desdobramentos em planos e programas integrem as questões ambientais e de sustentabilidade (White \& Noble, 2013).

Em 2010, foi instituída a PNRS, que reúne princípios, objetivos, instrumentos, diretrizes, metas e ações a serem adotados pela União de maneira isolada ou em parceria com Estados, Distrito Federal, municípios e particulares, visando à gestão ambientalmente adequada dos resíduos sólidos. Essa lei define as responsabilidades do poder público e dos geradores e impõe obrigações aos Estados e municípios. Entre 
seus pontos relevantes, destacam-se: a) encerramento dos vazadouros a céu aberto (lixões) e destinação final adequada dos rejeitos até 2014 - na data da elaboração deste artigo, esse prazo estava sendo revisto de acordo com o Projeto de lei no 2.289/15, postergando esse encerramento de 2018 a 2021, a depender das características e do porte do município; b) elaboração de acordos setoriais envolvendo toda a cadeia de geração e consumo com o objetivo de implementar a responsabilidade compartilhada pelo ciclo de vida dos produtos e a logística reversa; c) elaboração de planos nacional, estaduais e municipais de resíduos sólidos; d) conteúdo mínimo para o Plano de Gestão Integrada de Resíduos Sólidos (PGIRS) (Brasil, 2010). O PNRS encontra-se em revisão desde 2017 para estabelecimento de novas metas em função de algumas distorções do diagnóstico considerado no plano original. Essas metas têm impacto direto nas metas dos planos das esferas administrativas subsequentes.

Para Jacobi \& Besen (2011, p. 135), “[...] a PNRS fortalece os princípios da gestão integrada e sustentável de resíduos". Segundo a PNRS, o PGIRS deve incluir metas de redução, reutilização, coleta seletiva e reciclagem visando à redução da disposição final de resíduos. Também deve prever a participação do poder público local na coleta seletiva, na logística reversa e em outras ações relacionadas à responsabilidade compartilhada pelo ciclo de vida dos produtos; a identificação dos passivos ambientais relacionados aos resíduos sólidos e suas respectivas medidas; e o programa de monitoramento e ações preventivas e corretivas (Oliveira \& Galvão, 2016). 0 gerenciamento integrado de resíduos sólidos urbanos é constituído por um conjunto de instrumentos e técnicas que devem ser aplicados pelo município visando ao aumento da eficiência na gestão (Espinosa \& Silvas, 2014).

Cabe aos municípios a realização do PGIRS e sua integração aos planos que versem sobre questões relacionadas à gestão de resíduos sólidos, como o plano diretor, o plano de saneamento básico, o plano de mudanças climáticas, o plano de consórcios públicos, além do respeito à hierarquia dos instrumentos de planejamento de resíduos sólidos em todos os níveis da federação, conforme mostra a Figura 1 (nível nacional, estadual, microrregional, metropolitano, intermunicipal e municipal) (Brasil, 2012).

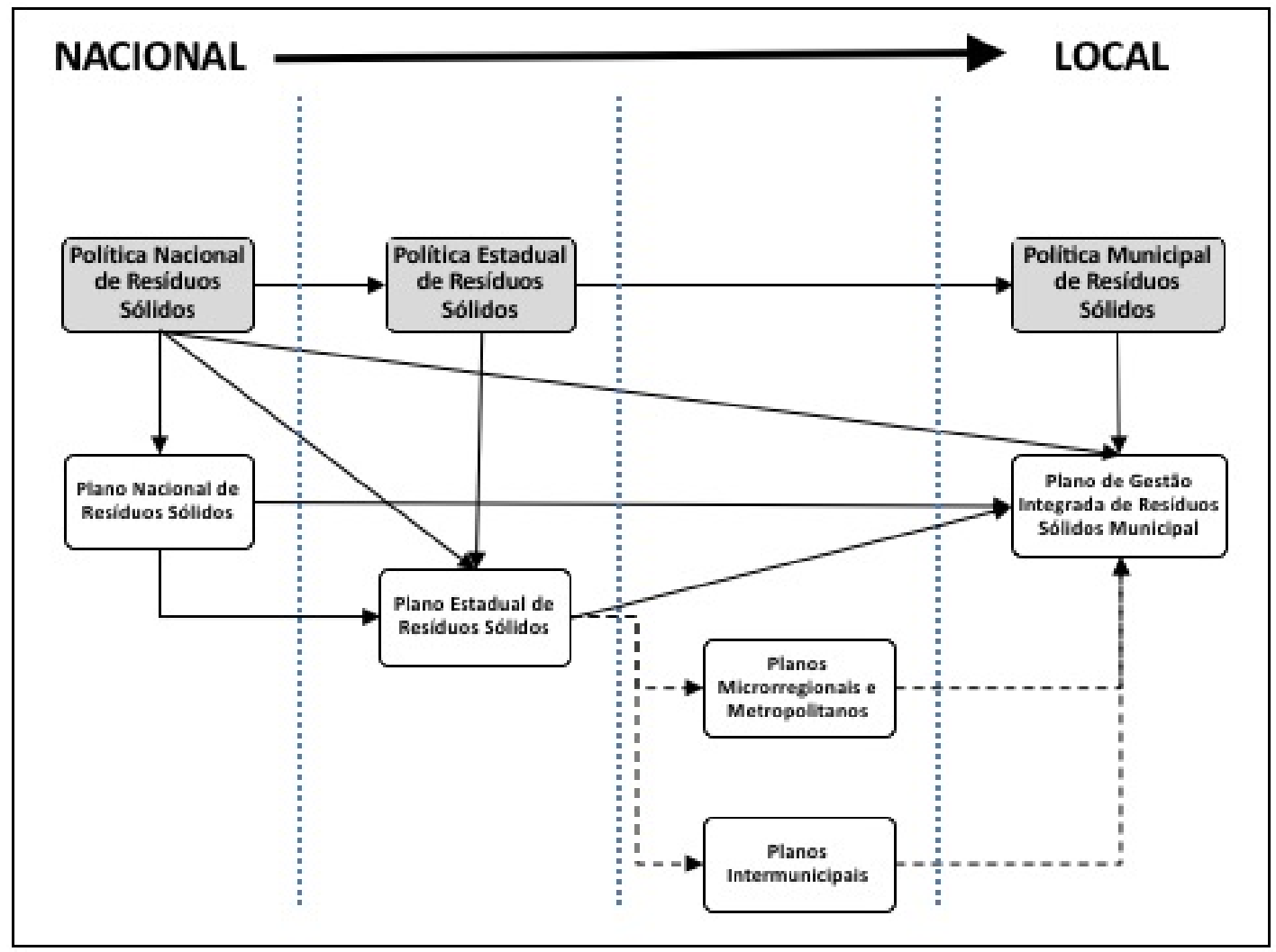

Figura 1 - Hierarquia entre os instrumentos de planejamento e gestão de resíduos sólidos no Brasil. Fonte: Elaborada pelos autores com base em Brasil (2012). 
A expectativa do sistema é que as políticas de resíduos sólidos dos níveis mais locais sejam alimentadas pelas políticas estabelecidas nos níveis mais abrangentes. A lógica também é que cada política estabeleça os critérios básicos para os planos. Na Figura 1, os níveis regionais e metropolitanos aparecem pontilhados porque não há políticas específicas, e nem sempre haverá planos desse tipo envolvendo todos os municípios. No entanto, esses planos poderiam ocorrer desde que a Política Nacional de Meio Ambiente e a Política Estadual de Meio Ambiente de São Paulo incluíssem o incentivo à solução conjunta em municípios vizinhos e regiões. A PNRS está influenciando os planos estaduais e municipais porque possui critérios para ambos os níveis. 0 planejamento do PNRS deve permitir que seus objetivos sejam alcançados por cada ente federado e pelos geradores de resíduos. Embora o conjunto de planos apresentados na Figura 1 esteja presente no escopo da referida lei, cada ente possui liberdade para executar seu plano, sob a influência da hierarquização da tomada de decisões sobre metas e ações determinadas pelo plano nacional (Oliveira \& Galvão, 2016). De acordo com Malvestio et al. (2012), esse modelo top-down de planejamento, em que as esferas superiores orientam as inferiores, é recorrente, permitindo um encadeamento na tomada de decisão em que cada nível administrativo possa estar direcionado às questões pertinentes ao seu nível decisório pelos documentos técnicos do planejamento. 0 plano municipal, ou intermunicipal, quando este o substitui, precisa ser a base dos planos de gerenciamento de resíduos daqueles geradores que têm a obrigação de elaborar e implantar seus próprios planos.

O Estado de São Paulo também possui uma política de resíduos sólidos. O plano estadual não estabelece ações para os municípios, cujo gerenciamento é de competência municipal, mas propõe metas a serem cumpridas no Estado em relação à disposição final e estimula soluções intermunicipais, bem como determina o fomento a boas práticas nos municípios e as diretrizes para financiamento de projetos dessa natureza por parte do Estado.

\section{Avaliação Ambiental Estratégica e planejamento de resíduos sólidos}

A AAE é um instrumento de inserção da variável ambiental na tomada de decisão naqueles estágios decisórios do planejamento que antecedem os projetos de engenharia, em nível de políticas, planos e programas (PPPs) (Rizzo et al., 2017). No Brasil, pode ter seu uso considerado restrito (Montaño et al., 2014), uma vez que não há obrigatoriedade para sua aplicação (Gallardo et al., 2016), e são poucas as experiências reconhecidas da utilização da AAE no planejamento brasileiro (Nadruz et al., 2018). A AAE visa avaliar a viabilidade das opções estratégicas considerando-se questões ambientais e de sustentabilidade, de modo a possibilitar o alcance dos macro-objetivos do planejamento (Partidário, 2012), como é o caso da PNRS.

De acordo com Tang (2008), um dos principais problemas no planejamento municipal reside na falta de integração do processo de planejamento com o de tomada de decisão, uma vez que esse nível decisório não tem o potencial para incorporar os impactos ambientais críticos em escalas temporal e espacial amplas, lacuna que pode ser suprimida pelo uso da AAE. A AAE possibilita essa análise integrada de modo a permitir que os objetivos estratégicos estejam contemplados nos níveis decisórios operacionais ou da base da pirâmide de decisão (Arts et al., 2011).

Segundo Desmond (2009), pelo tiering (hierarquização ou encadeamento) as decisões da política estabelecem um contexto para as decisões subsequentes, gerando uma integração nessa trajetória do planejamento em diferentes níveis de poder, do mais elevado (políticas, por exemplo) para o nível local (como um plano municipal). Para Turco \& Gallardo (2018, p. 5), “[...] o nível superior decisório deve influenciar o nível subsequente", permitindo que objetivos ambientais estratégicos possam acompanhar o processo de tomada de decisão até o nível de projeto ou nível local de intervenção (tiering vertical). Arts et al. (2011) também reconhecem o tiering horizontal, que se caracteriza pela influência entre planos de diferentes setores em um mesmo nível estratégico, e o tiering diagonal, marcado pela combinação entre o tiering vertical e o tiering horizontal. 
Fischer et al. (2011) analisaram as estratégias de 25 planos municipais de gestão de resíduos da Inglaterra e verificaram que a AAE proporcionou atingir práticas de gerenciamento com foco na hierarquia da gestão de resíduos. Por sua vez, Josimović et al. (2015) mostraram o uso de avaliação multicritério aplicada à AAE para aprimorar a gestão de resíduos em Belgrado. Por outro lado, Salhofer et al. (2007) destacaram que a AAE empregada para desenvolver o plano regional de resíduos em Salzburgo direcionou-se à discussão de alternativas, sem apontar a melhor solução, gerando dificuldades em lidar com um grande número de pequenos resultados e facilidades de entender que a AAE é um processo que remete à aprendizagem e promove uma ativa participação pública e a formulação de cenários futuros otimizados.

Os procedimentos mais discutidos para realização da AAE seguramente são os desenvolvidos a partir da Diretiva Europeia de AAE (EC, 2011), que promulgou sua obrigatoriedade no planejamento em nível de planos e programas nos países da União Europeia (Partidario \& Sheate, 2013). Vários trabalhos detalham esses procedimentos, como os de Partidário (2012), McGimpsey \& Morgan (2013) e Gallardo et al. (2016), revelando similaridades entre as sequências lógicas do processo e das atividades técnicas.

A AAE apresenta-se como uma abordagem para a avaliação integrada no planejamento de resíduos sólidos que envolve a participação social, um dos elemento-chave do processo de AAE, pois traz transparência e auxilia na redução de conflitos na tomada de decisão (Salhofer et al., 2007). Para Desmond (2009), a AAE nesse contexto pode catalisar a aplicação da legislação de gestão de resíduos sólidos. Segundo Malvestio et al. (2012), o planejamento proposto pela PNRS apresenta um formato parecido ao de uma AAE, uma vez que a política estabelece várias etapas na formulação do plano que estão também presentes em etapas da AAE.

\section{Método}

Esta pesquisa qualitativa de objetivo exploratório foi conduzida por meio de pesquisa bibliográfica e pesquisa documental, a partir de um estudo de caso, o PGIRS de São Paulo.

\section{Unidade de análise}

O município de São Paulo contava, no último censo de 2010, com uma população de 11.253.503 habitantes, estimada para 2017 em 12.106.920 pessoas (IBGE, 2018), e, em 2012, coletou em média $20.100 \mathrm{t} /$ dia de resíduos sólidos urbanos, possuindo uma geração média per capita de $1,1 \mathrm{~kg} / \mathrm{hab} /$ dia (São Paulo, 2014). Aproximadamente 51\% dos resíduos sólidos residenciais coletados em São Paulo são orgânicos e dispostos em aterros do município, tanto encerrados como em operação, gerando $14 \%$ de todos os gases de efeito estufa emitidos no município (São Paulo, 2014). Em cumprimento à PNRS, em 2014 São Paulo lançou o PGIRS. A primeira versão do plano foi oficializada em 2012 e revisada em 2014, por não atender a requisitos mínimos da PNRS. A responsabilidade pelo plano foi da Secretaria Municipal de Serviços, segundo a qual a reelaboração do plano contou com forte participação popular durante a IV Conferência Municipal de Meio Ambiente (São Paulo, 2014).

\section{Coleta e análise de dados}

A pesquisa bibliográfica pautou-se no levantamento de referenciais de boas práticas de AAE, apoiadas nas diretrizes da Diretiva Europeia de AAE (EC, 2011), principal e mais testado referencial técnico sobre o instrumento, como os trabalhos de Partidário (2012), McGimpsey \& Morgan (2013) e Gallardo et al. (2016), e de referenciais que permitissem entender o tiering no contexto da AAE e da PNRS.

Para analisar a adequação do PGIRS de São Paulo às orientações internacionais de boas práticas de AAE, optou-se pelo referencial de análise proposto por Partidário (2012), visto que se trata de um roteiro 
pautado nas melhores práticas de AAE. A análise de como os objetivos estratégicos da PNRS influenciam o PGIRS deu-se a partir de dois enfoques: a) com base no referencial de Malvestio et al. (2012), analisouse o enfoque teórico esperado para planos municipais e para AAE desses planos comparado ao PGIRS; b) com base no referencial de Desmond (2009), analisou-se o tiering desde a política federal até o plano municipal, tendo como foco a hierarquia da gestão de resíduos sólidos.

0 estudo documental considerou o PGIRS de São Paulo (2014), que está apresentado em 456 páginas, distribuídas em 15 seções, e a Lei de Política Nacional de Resíduos Sólidos (Brasil, 2010), que está descrita em 76 páginas, com 4 títulos principais.

A análise dos documentos buscou categorizar os trechos de interesse dentro de categorias específicas, por meio de análise de conteúdo (Bardin, 2009). As dimensões de análise foram: a) análise do conteúdo do PGIRS (São Paulo, 2014), de acordo com as diretrizes de boas práticas de AAE propostas por Partidário (2012) e os objetivos da AAE discutidos por Malvestio et al. (2012); b) análise das estratégias, diretrizes e metas do PGIRS quanto aos princípios da PNRS, dentro do conceito de tiering em planejamento, conforme proposto por Desmond (2009).

\section{As boas práticas de AAE e o PGIRS de São Paulo}

O conteúdo do PGIRS de São Paulo foi comparado às boas práticas de AAE estabelecidas por Partidário (2012), conforme apresentado no Quadro 1.

Quadro 1 - PGIRS de São Paulo e boas práticas de AAE

\begin{tabular}{|c|c|c|c|}
\hline $\begin{array}{l}\text { Elementos- } \\
\text { chave da AAE }\end{array}$ & Perguntas-chave & Boas práticas de $\mathrm{AAE}$ & $\begin{array}{l}\text { Análise do conteúdo do PGIRS de } \\
\text { São Paulo }\end{array}$ \\
\hline $\begin{array}{l}\text { 1. Objeto da } \\
\text { avaliação. }\end{array}$ & $\begin{array}{l}\text { O que foi } \\
\text { avaliado? }\end{array}$ & $\begin{array}{l}\text { A AAE deve apresentar a } \\
\text { identificação de cenários e/ou } \\
\text { alternativas estratégicas. }\end{array}$ & $\begin{array}{l}\text { No PGIRS, foram considerados } \\
\text { cenários e opções estratégicas } \\
\text { para gerenciamento de resíduos. }\end{array}$ \\
\hline 2. Início da AAE. & $\begin{array}{l}\text { Em que fase se } \\
\text { iniciou a AAE no } \\
\text { processo de } \\
\text { planejamento? }\end{array}$ & $\begin{array}{l}\text { A AAE é aplicada consoante o } \\
\text { desenvolvimento do plano. }\end{array}$ & $\begin{array}{l}\text { Não se aplica. A AAE não foi } \\
\text { utilizada durante o } \\
\text { desenvolvimento do PGIRS. }\end{array}$ \\
\hline 3. Interatividade. & $\begin{array}{l}\text { Qual foi o grau de } \\
\text { integração e de } \\
\text { retorno entre as } \\
\text { atividades de } \\
\text { avaliação e } \\
\text { planejamento? }\end{array}$ & $\begin{array}{l}\text { A AAE deve garantir que a } \\
\text { integração entre atividades de } \\
\text { avaliação e planejamento seja } \\
\text { elevada. }\end{array}$ & $\begin{array}{l}\text { De acordo com o PGIRS, o Decreto } \\
\text { n 53.924/13 criou o Comitê } \\
\text { Intersecretarial para a Política } \\
\text { Municipal de Resíduos Sólidos, } \\
\text { composto de várias secretarias, } \\
\text { integrando diferentes setores. }\end{array}$ \\
\hline $\begin{array}{l}\text { 4. Quadro- } \\
\text { problema. }\end{array}$ & $\begin{array}{l}\text { Foram identificados } \\
\text { problemas? }\end{array}$ & $\begin{array}{l}\text { A AAE deve apresentar um } \\
\text { mapeamento sintético e } \\
\text { circunscrito dos principais } \\
\text { problemas, das potencialidades e } \\
\text { das forças motrizes que } \\
\text { representam as prioridades } \\
\text { setoriais, ambientais e de } \\
\text { sustentabilidade. }\end{array}$ & $\begin{array}{l}\text { Foi realizado no PGIRS um } \\
\text { diagnóstico da situação atual dos } \\
\text { resíduos sólidos no município, de } \\
\text { acordo com os aspectos } \\
\text { socioeconômicos, a composição } \\
\text { dos resíduos gerados e coletados e } \\
\text { as atuais áreas de destinação e } \\
\text { disposição, identificando os } \\
\text { problemas de gestão de resíduos. }\end{array}$ \\
\hline $\begin{array}{l}\text { 5. Quadro de } \\
\text { governança. }\end{array}$ & $\begin{array}{l}\text { Foi estabelecido } \\
\text { um quadro de } \\
\text { governança? }\end{array}$ & $\begin{array}{l}\text { A AAE deve envolver } \\
\text { responsabilidade institucional, } \\
\text { cooperação institucional e } \\
\text { envolvimento de agentes } \\
\text { interessados. Para tanto, deve criar } \\
\text { uma rede de instituições, } \\
\text { organizações governamentais e } \\
\text { não governamentais e painéis de } \\
\text { cidadão, e incluir princípios de } \\
\text { responsabilidade, transparência, } \\
\text { integridade, eficiência e liderança } \\
\text { para o desempenho efetivo do } \\
\text { planejamento. }\end{array}$ & $\begin{array}{l}\text { De acordo com o PGIRS, o Comitê } \\
\text { Intersecretarial para a Política } \\
\text { Municipal de Resíduos Sólidos } \\
\text { congrega secretarias, setores e } \\
\text { grupos de trabalho. Os grupos de } \\
\text { trabalho contêm representantes da } \\
\text { sociedade civil, porém não } \\
\text { abrangem todos os atores } \\
\text { esperados em um quadro de } \\
\text { governança e tampouco } \\
\text { preenchem todos os requisitos } \\
\text { esperados. }\end{array}$ \\
\hline
\end{tabular}


Quadro 1 -Continuação...

\begin{tabular}{|c|c|c|c|}
\hline $\begin{array}{l}\text { Elementos- } \\
\text { chave da AAE }\end{array}$ & Perguntas-chave & Boas práticas de AAE & $\begin{array}{l}\text { Análise do conteúdo do PGIRS de } \\
\text { São Paulo }\end{array}$ \\
\hline $\begin{array}{l}\text { 6. Quadro } \\
\text { estratégico de } \\
\text { referência. }\end{array}$ & $\begin{array}{l}\text { Como foi o quadro } \\
\text { de referência } \\
\text { estratégico } \\
\text { estabelecido? }\end{array}$ & $\begin{array}{l}\text { A AAE deve incluir objetivos e } \\
\text { metas de políticas ambientais e de } \\
\text { sustentabilidade (até 30) para } \\
\text { definir o referencial de avaliação } \\
\text { estratégica. }\end{array}$ & $\begin{array}{l}\text { No PGIRS, não foi estabelecido um } \\
\text { quadro de referência estratégico } \\
\text { que seria representado por } \\
\text { objetivos e metas de políticas } \\
\text { estratégicas nacionais para níveis } \\
\text { decisórios institucionais e outras } \\
\text { políticas aplicáveis com sinergias e } \\
\text { conflitos com a analisada. O único } \\
\text { referencial adotado é a própria } \\
\text { PNRS. }\end{array}$ \\
\hline $\begin{array}{l}\text { 7. Quadro de } \\
\text { avaliação de } \\
\text { fatores críticos } \\
\text { para a tomada } \\
\text { de decisão. }\end{array}$ & $\begin{array}{l}\text { O quadro de } \\
\text { avaliação a partir } \\
\text { de fatores críticos- } \\
\text { são temas-chave } \\
\text { integrados que } \\
\text { estabelecem o } \\
\text { foco da AAE, a } \\
\text { estrutura de } \\
\text { avaliação e o } \\
\text { conteúdo dos } \\
\text { estudos técnicos- } \\
\text { para a tomada de } \\
\text { decisão foi bem } \\
\text { definido? }\end{array}$ & $\begin{array}{l}\text { Esse quadro deve ser holístico e } \\
\text { integrado. } \\
\text { Apresentação de poucos fatores } \\
\text { críticos de decisão, porém } \\
\text { integrados. }\end{array}$ & $\begin{array}{l}\text { Não foi estabelecido. Mas, a partir } \\
\text { do PGIRS, podem ser reconhecidos } \\
\text { vários fatores relevantes: gestão de } \\
\text { resíduos; inovação, conhecimento } \\
\text { e conscientização ambiental; } \\
\text { qualidade ambiental e espaço } \\
\text { urbano; geração de emprego e } \\
\text { renda; recursos naturais; recursos } \\
\text { financeiros. Porém, isso não denota } \\
\text { um foco integrado e não há uma } \\
\text { análise holística desses fatores. }\end{array}$ \\
\hline $\begin{array}{l}\text { 8. Opções } \\
\text { estratégicas. }\end{array}$ & $\begin{array}{l}\text { Foram avaliadas } \\
\text { opções } \\
\text { estratégicas, que } \\
\text { são alternativas } \\
\text { que garantam que } \\
\text { o planejamento } \\
\text { englobe questões } \\
\text { ambientais e de } \\
\text { sustentabilidade, } \\
\text { conforme os } \\
\text { objetivos do plano? }\end{array}$ & $\begin{array}{l}\text { A AAE deve considerar as } \\
\text { oportunidades e os riscos das } \\
\text { opções para as estratégias } \\
\text { relevantes consideradas no escopo } \\
\text { do plano. }\end{array}$ & $\begin{array}{l}\text { O PGIRS define um conjunto de } \\
\text { estratégias para cada tipo de } \\
\text { resíduos gerados no município, } \\
\text { bem como a definição de metas, } \\
\text { prazos, custos e competências. }\end{array}$ \\
\hline 9. Participação. & $\begin{array}{l}\text { Qual foi o grau de } \\
\text { participação? } \\
\text { Deve-se avaliar os } \\
\text { mecanismos } \\
\text { empregados para } \\
\text { o envolvimento dos } \\
\text { cidadãos na } \\
\text { decisão. }\end{array}$ & $\begin{array}{l}\text { A AAE deve possibilitar elevado } \\
\text { envolvimento das partes } \\
\text { interessadas. Processo ativo de } \\
\text { participação. }\end{array}$ & $\begin{array}{l}\text { O processo participativo de } \\
\text { concepção do PGIRS envolveu } \\
\text { mais de } 7 \text { mil pessoas em } \\
58 \text { eventos, incluindo } 31 \text { pré- } \\
\text { conferências, } 6 \text { reuniões temáticas, } \\
9 \text { reuniões preparatórias dos } \\
800 \text { delegados, } 10 \text { oficinas técnicas } \\
\text { com cadeias produtivas para } \\
\text { proposição de metas, além de } \\
\text { uma conferência municipal. } \\
\text { Entretanto, não houve um sistema } \\
\text { de divulgação contínua das } \\
\text { informações coletadas. }\end{array}$ \\
\hline $\begin{array}{l}\text { 10. Diretrizes para } \\
\text { o planejamento, } \\
\text { a gestão e o } \\
\text { monitoramento. }\end{array}$ & $\begin{array}{l}\text { Existem diretrizes } \\
\text { para o } \\
\text { acompanhamento } \\
\text { da implementação } \\
\text { do plano? }\end{array}$ & $\begin{array}{l}\text { A AAE deve estabelecer diretrizes } \\
\text { para o planejamento, a gestão e o } \\
\text { monitoramento, devendo } \\
\text { incluir até } 20 \text { indicadores de } \\
\text { monitoramento. }\end{array}$ & $\begin{array}{l}\text { O PGIRS apresenta diretrizes para } \\
\text { acompanhamento, porém não } \\
\text { apresenta indicadores. Mas há } \\
\text { orientações para a criação de } \\
\text { indicadores de monitoramento das } \\
\text { ações, dos programas e das metas } \\
\text { previstos. }\end{array}$ \\
\hline
\end{tabular}

Fonte: Elaborado pelos autores com base em Partidário (2012).

Pela análise do Quadro 1, considera-se que o PGIRS abrangeu tópicos do planejamento subsidiado pela AAE para a gestão dos resíduos em âmbito municipal quanto: à interatividade entre atividades de avaliação 
e planejamento (conferência e comitês integrando diversos setores); ao mapeamento da situação ambiental (diagnóstico da geração de resíduos sólidos e identificação de problema de manejo e criação de cenários futuros); às estratégias, às metas, aos prazos, aos custos e às competências (conjunto de estratégias para cada tipo de resíduo sólido urbano). Alguns elementos de boas práticas de AAE, como participação pública e quadro de governança, estavam presentes, mas poderiam ser mais bem explorados. 0 quadro de governança poderia ser ampliado para além dos atores envolvidos, e a participação pública envolveu diversos setores, comitês e grupos de trabalho, mas não houve um sistema de informações e divulgação sistemática. As principais limitações quanto às boas práticas de AAE referem-se: à falta de delimitação objetiva dos fatores críticos à decisão; à ausência do sistema de informações sem a proposição de uma cesta de indicadores para avaliação sistemática do plano e de suas revisões futuras; à falta de um quadro de referências para além daquelas intrínsecas ao setor de resíduos. Apesar da ausência da provisão de indicadores, um aspecto positivo do PGIRS diz respeito à apresentação das diretrizes para a constituição de um sistema de informações sobre a gestão de resíduos sólidos, o SIMIRS-SP, o que é considerado essencial na AAE, como preconizado por Partidário (2012).

Embora tenham sido realizadas dez oficinas técnicas com cadeias produtivas, considera-se que a participação pública do setor privado se limitou à discussão das metas. A interação entre a estrutura pública e o setor privado tem sido apontada como um aspecto importante da gestão de resíduos. No caso dos planos de gestão municipal de resíduos, eles interagem diretamente por meio dos planos dos geradores e com os mecanismos de mercado que podem contribuir para a logística reversa, gestão de ciclo de vida de produtos e outras ferramentas avançadas propostas pelas PNRS. Certos aspectos de competência municipal, mas regulados em outros níveis de governo, como a logística reversa, têm papel imprescindível ao setor privado para sucesso. Pelo PGIRS (São Paulo, 2014), não está evidente como e com qual intensidade ocorreu a participação do setor privado nas oficinas e nos eventos técnicos, o que dificulta avaliar a capacidade do PGIRS de abarcar soluções mais complexas envolvendo o setor privado, destacadas por Zanta \& Ferreira (2003) e UNEP (2011). A participação pública proporcionada pela AAE, em que a representação justa das partes interessadas é um fator de sucesso (Rega \& Baldizzone, 2015), permite garantir transparência e aceitação do processo decisório (Salhofer et al., 2007), identificando uma relação positiva entre o grau de envolvimento público e o desempenho do plano. 0 quadro de governança na AAE, de acordo com Monteiro \& Partidário (2017), promove engajamento e possibilita ações colaborativas e processos de aprendizagem, alcançando elevado nível das considerações ambientais no planejamento, sendo, assim, fundamental para a implementação de um plano de resíduos sólidos.

A Figura 2 compara o enfoque do planejamento estabelecido pela PNRS com o enfoque que a abordagem da AAE remete ao planejamento, conforme discutido por Malvestio et al. (2012), aplicados ao conteúdo do PGIRS de São Paulo (2014). 


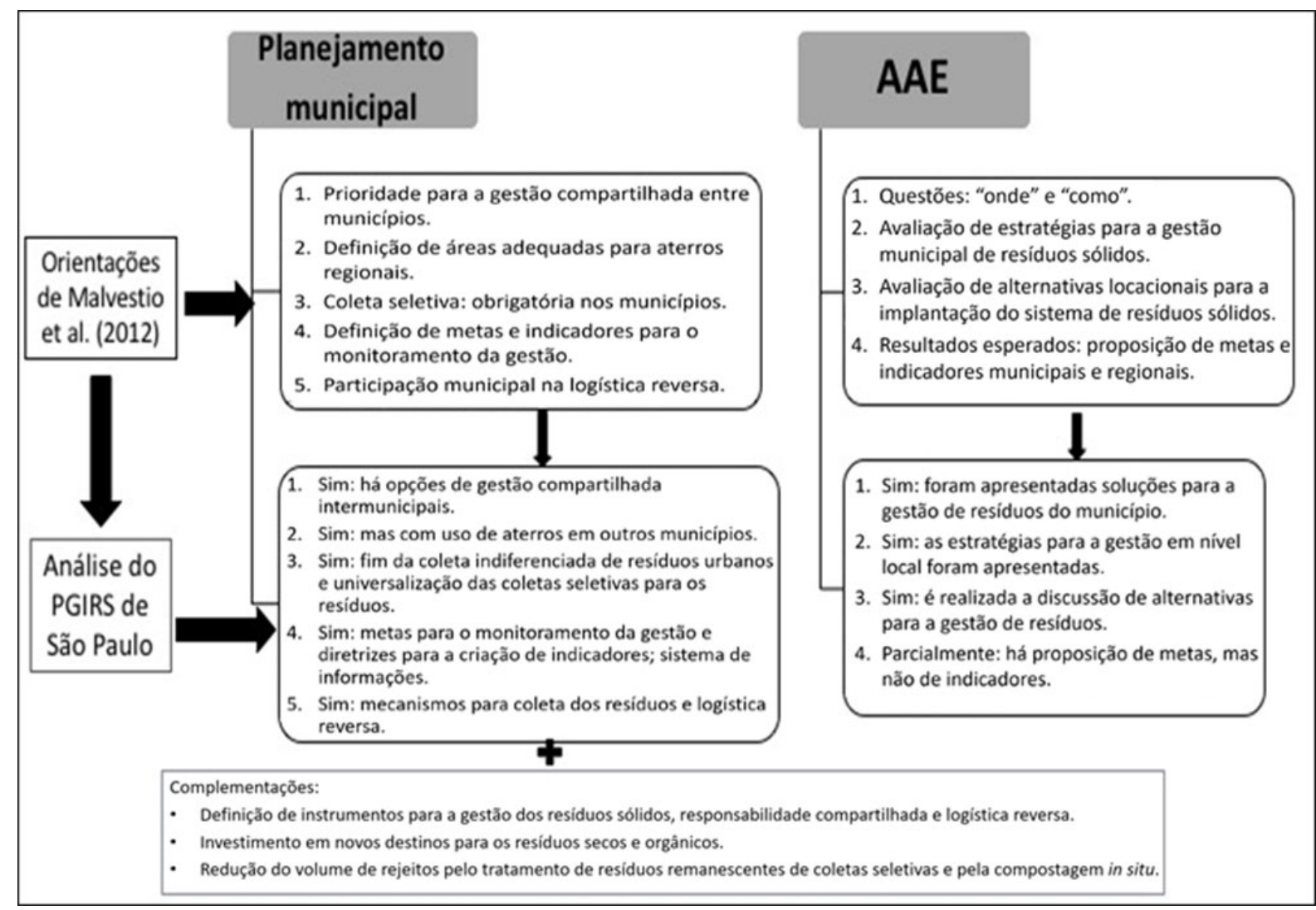

Figura 2 - Comparação dos enfoques do planejamento e da AAE esperados para plano municipal, aplicados ao conteúdo do PGIRS de São Paulo. Fonte: Elaborada pelos autores com base em Malvestio et al. (2012).

Pela análise da Figura 2, observa-se que o conteúdo do PGIRS está mais abrangente do que o conteúdo mínimo esperado ao escopo do plano de resíduos em nível municipal, descrito por Malvestio et al. (2012), e apresenta um bom alinhamento com o conteúdo da AAE, também apresentado por Malvestio et al. (2012), à exceção da ausência dos indicadores.

A presença de indicadores é uma das boas práticas da AAE (Partidário, 2012). Devem ser usados para demonstrar as mudanças na qualidade ambiental resultantes da implementação de planos e fornecer informações que permitam atender às metas estabelecidas pela AAE (Donnelly et al., 2007). 0 uso de indicadores globais de eficácia do processo é uma demanda para implementar a gestão de longo prazo. Silva et al. (2017) avaliaram o desempenho da gestão de resíduos em Curitiba por meio de indicadores quantitativos, como porcentagem de resíduos coletados e reciclados, geração de resíduos por habitante, custo global da limpeza pública e proporção de origem de recursos entre receita e recursos do orçamento público. A avaliação da gestão dos resíduos em médio e longo prazo, assim como sua comparação aos resultados de outras cidades, fica prejudicada na ausência desses indicadores. 0 uso de indicadores permite propor ajustes no plano com ações corretivas, conforme recomendação de Oliveira \& Galvão (2016), subsidiar avaliação multicritério na gestão de resíduos em Belgrado, de acordo com o relato de Josimović et al. (2015), e ainda afastar as dificuldades de avaliação dos sistemas de gestão de resíduos em Salzburgo, apontadas por Salhofer et al. (2007).

Pelo Figura 2, observa-se um alinhamento do PGIRS às boas práticas de AAE e não se verificam situações em que os objetivos da AAE se mostram conflitantes com os do PGIRS, confirmando o potencial de aplicação do instrumento a esse planejamento setorial e reforçando os achados de Fischer et al. (2011), Partidário (2012), Josimović et al. (2015), Desmond (2009) e Malvestio et al. (2012). Além disso, pode-se auferir os benefícios da prática da AAE discutidos por Sánchez \& Silva-Sánchez (2008), Montaño et al. (2014), Gallardo et al. (2016), Rizzo et al. (2017) e Nadruz et al. (2018). Nota-se no PGIRS o incentivo às ações de fomento ao conhecimento, à educação e à conscientização ambiental em matéria 
de resíduos sólidos, apresentando nítida compatibilidade com os objetivos intrínsecos às boas práticas de AAE (Tetlow \& Hanusch, 2012).

\section{Tiering no planejamento de resíduos sólidos: da PNRS ao PGIRS}

O Quadro 2 mostra a perspectiva de tiering no planejamento de gestão de resíduos sólidos no Brasil em suas esferas decisórias, incluindo as etapas de gerenciamento (Philippi \& Aguiar, 2018; Fischer et al., 2011), conforme destacado por Desmond (2009), com o foco no PGIRS de São Paulo.

Quadro 2 - Avaliação do tiering por meio das opções de gestão de resíduos sólidos na hierarquia de resíduos, desde a PNRS até o PGIRS de São Paulo

\begin{tabular}{|c|c|c|c|c|}
\hline $\begin{array}{l}\text { Nível: política } \\
\text { nacional / } \\
\text { estratégia }\end{array}$ & $\begin{array}{l}\text { Nível: planejamento } \\
\text { regional }\end{array}$ & $\begin{array}{c}\text { Nível: planejamento } \\
\text { local }\end{array}$ & $\begin{array}{l}\text { Nível: programa/ } \\
\text { projeto }\end{array}$ & PGIRS de São Paulo \\
\hline $\begin{array}{l}\text { Alternativas políticas } \\
\text { (hierarquia de } \\
\text { resíduos). }\end{array}$ & $\begin{array}{l}\text { Opções de gestão } \\
\text { de resíduos. }\end{array}$ & $\begin{array}{l}\text { Opções de } \\
\text { localização. }\end{array}$ & $\begin{array}{l}\text { Implementação / } \\
\text { opções temporais. }\end{array}$ & $\begin{array}{l}\text { Todos esses } \\
\text { elementos são } \\
\text { observados no } \\
\text { PGIRS e discutidos } \\
\text { para o nível de } \\
\text { ação municipal. }\end{array}$ \\
\hline $\begin{array}{l}\text { Minimização de } \\
\text { resíduos. }\end{array}$ & $\begin{array}{l}\text { Prevenção e } \\
\text { minimização. }\end{array}$ & $\begin{array}{l}\text { Escritórios, casas e } \\
\text { indústria. }\end{array}$ & $\begin{array}{l}\text { Implementação de } \\
\text { programas e } \\
\text { projetos de } \\
\text { minimização de } \\
\text { resíduos. }\end{array}$ & $\begin{array}{l}\text { Plano municipal de } \\
\text { educação } \\
\text { ambiental voltado } \\
\text { para a gestão de } \\
\text { resíduos sólidos. }\end{array}$ \\
\hline Reutilização. & $\begin{array}{l}\text { Prolongamento da } \\
\text { vida útil do produto. }\end{array}$ & Bens retornáveis. & $\begin{array}{l}\text { Implementação de } \\
\text { programas de } \\
\text { reutilização de } \\
\text { resíduos. }\end{array}$ & $\begin{array}{l}\text { Implantação de } \\
\text { programas de } \\
\text { logística reversa e } \\
\text { outras formas de } \\
\text { reutilização. }\end{array}$ \\
\hline \multirow{2}{*}{$\begin{array}{l}\text { Recuperação e } \\
\text { reciclagem. }\end{array}$} & Opções de coleta. & $\begin{array}{l}\text { Instalação de } \\
\text { recuperação de } \\
\text { materiais (MRF). }\end{array}$ & $\begin{array}{l}\text { Detalhes específicos } \\
\text { de rotas, horários, } \\
\text { local e gestão. }\end{array}$ & $\begin{array}{l}\text { Programas de } \\
\text { coleta seletiva, } \\
\text { pontos de entrega } \\
\text { voluntária (PEVs) e } \\
\text { ecopontos para a } \\
\text { entrega dos resíduos } \\
\text { sólidos recicláveis } \\
\text { secos. }\end{array}$ \\
\hline & & $\begin{array}{l}\text { Opções de } \\
\text { compostagem. } \\
\text { Processos de } \\
\text { estabilização e TMB. } \\
\text { Processo com base } \\
\text { em RDF } \\
\text { (combustível } \\
\text { derivado de } \\
\text { resíduos). }\end{array}$ & $\begin{array}{l}\text { Escolha do processo } \\
\text { de instalação de } \\
\text { recuperação de } \\
\text { materiais (MRF). }\end{array}$ & $\begin{array}{l}\text { Definidos programas } \\
\text { de valorização de } \\
\text { resíduos orgânicos - } \\
\text { compostagem e } \\
\text { recuperação } \\
\text { energética. }\end{array}$ \\
\hline \multirow{2}{*}{$\begin{array}{l}\text { Recuperação e } \\
\text { reciclagem. }\end{array}$} & Recicláveis secos. & $\begin{array}{l}\text { Incineração. } \\
\text { Aterro. } \\
\text { Reciclagem. }\end{array}$ & & $\begin{array}{l}\text { Iniciativas de } \\
\text { gerenciamento dos } \\
\text { recicláveis secos e } \\
\text { incineração dos } \\
\text { resíduos de serviço } \\
\text { de saúde. }\end{array}$ \\
\hline & $\begin{array}{l}\text { Opções de } \\
\text { tratamento de } \\
\text { orgânicos. }\end{array}$ & & $\begin{array}{l}\text { Opções de } \\
\text { compostagem. }\end{array}$ & $\begin{array}{l}\text { Para a gestão e o } \\
\text { manejo dos resíduos } \\
\text { orgânicos, o plano } \\
\text { prevê diversas } \\
\text { ações. }\end{array}$ \\
\hline
\end{tabular}


Quadro 2 -Continuação...

\begin{tabular}{|c|c|c|c|c|}
\hline $\begin{array}{l}\text { Nível: política } \\
\text { nacional / } \\
\text { estratégia }\end{array}$ & $\begin{array}{l}\text { Nível: planejamento } \\
\text { regional }\end{array}$ & $\begin{array}{c}\text { Nível: planejamento } \\
\text { local }\end{array}$ & $\begin{array}{l}\text { Nível: programa/ } \\
\text { projeto }\end{array}$ & PGIRS de São Paulo \\
\hline \multirow{3}{*}{$\begin{array}{l}\text { Recuperação de } \\
\text { energia. }\end{array}$} & $\begin{array}{l}\text { Incineração com } \\
\text { recuperação de } \\
\text { energia. }\end{array}$ & & $\begin{array}{l}\text { Projeto / gestão de } \\
\text { instalações de } \\
\text { recuperação de } \\
\text { energia. }\end{array}$ & $\begin{array}{l}\text { Somente os resíduos } \\
\text { de serviço de saúde } \\
\text { e embalagens de } \\
\text { óleos lubrificantes e } \\
\text { materiais } \\
\text { contaminados são } \\
\text { encaminhados para } \\
\text { incineração. }\end{array}$ \\
\hline & $\begin{array}{l}\text { Resíduos de } \\
\text { combustível } \\
\text { derivado. }\end{array}$ & & & $\begin{array}{l}\text { Projetos de } \\
\text { instalações em } \\
\text { aterros sanitários } \\
\text { pela captação de } \\
\text { gases provenientes } \\
\text { dos aterros. } \\
\end{array}$ \\
\hline & $\begin{array}{l}\text { Aterro de utilização } \\
\text { de gás. }\end{array}$ & & & $\begin{array}{l}\text { Projeto para } \\
\text { instalação de } \\
\text { unidades TMB em } \\
\text { ecoparques, com } \\
\text { tratamento do } \\
\text { resíduo orgânico por } \\
\text { biodigestão } \\
\text { anaeróbia, } \\
\text { transformando-o em } \\
\text { biogás. }\end{array}$ \\
\hline \multirow[t]{2}{*}{ Disposição final. } & $\begin{array}{l}\text { Aterros sanitários de } \\
\text { resíduos não } \\
\text { tratados. }\end{array}$ & & $\begin{array}{l}\text { Projeto / gestão de } \\
\text { instalações de } \\
\text { recuperação de } \\
\text { energia. }\end{array}$ & $\begin{array}{l}\text { Instalaçães de } \\
\text { recuperação de } \\
\text { energia em aterros } \\
\text { sanitários, por meio } \\
\text { da captação de } \\
\text { gases provenientes } \\
\text { dos aterros. }\end{array}$ \\
\hline & Resíduos tratados. & & & $\begin{array}{l}\text { Prevê que os } \\
\text { resíduos de serviço } \\
\text { de saúde (RSS) } \\
\text { sejam } \\
\text { encaminhados para } \\
\text { tratamento. }\end{array}$ \\
\hline
\end{tabular}

Fonte: Elaborado pelos autores com base em Desmond (2009).

O Quadro 2 permite considerar evidências de tiering no planejamento de resíduos da escala nacional para a local, denotando encadeamento na gestão de resíduos definida pela PNRS e observada no PGIRS. Esse resultado difere do encontrado por Maiello et al. (2018, p. 46), que analisaram o plano municipal do Rio de Janeiro e constataram que "[...] o processo de institucionalização da gestão de resíduos sólidos na Região Metropolitana do Rio de Janeiro, tendo como marco a PNRS, é apenas incipiente".

Cabe ressaltar que há diversos temas que requerem articulação municipal com outros níveis de governo: para resíduos sujeitos à logística reversa, os acordos setoriais são estaduais ou nacionais; para materiais recicláveis, a legislação tributária base é estadual e tem sido considerada uma barreira à reciclagem; quanto a áreas para disposição final, seu licenciamento é geralmente de responsabilidade do órgão licenciador estadual.

Observa-se que o PGIRS atende a todas as opções apresentadas no modelo proposto em nível nacional, exceto a utilização da incineração com recuperação de energia. A cidade já teve incineradores, mais recentemente usados para tratamento de resíduos de serviços de saúde. Em 1993, houve uma tentativa de implantação de incinerador de resíduos domiciliares, que foi descartada por causa da resistência da comunidade e de problemas no licenciamento. Na cidade de São Paulo, há recuperação de energia somente por meio de projetos de instalações em aterros sanitários que captam os gases gerados. 
Há também projetos para instalação de unidades de tratamento mecânico-biológico em ecoparques por biodigestão anaeróbia, transformando os gases em biogás e biofertilizante. Essas propostas abrem perspectivas para o enfrentamento das mudanças climáticas, aspecto positivo do PGIRS, em contraposição à crítica de Fischer et al. (2011) aos planos municipais do Reino Unido, os quais não englobaram essas ações. Percebe-se um elevado grau de compatibilidade entre os objetivos, as diretrizes e as estratégias direcionados à gestão de resíduos sólidos na cidade, sendo as ações propostas compatíveis com as recomendações geralmente presentes na literatura sobre gerenciamento integrado de resíduos sólidos, como Philippi \& Aguiar (2018), Jacobi \& Besen (2011) e UNEP (2011).

Além das nítidas evidências do tiering vertical (Turco \& Gallardo, 2018), desde a PNRS até o PGIRS, observam-se alguns indícios de tiering horizontal e diagonal, materializados pela menção da Lei Federal de Saneamento Básico e da Política Nacional sobre Mudanças do Clima, denotando um potencial de articulação entre setores influenciando a execução do PGIRS e os seus resultados.

\section{Conclusões e recomendações}

Considera-se que o processo de planejamento de gestão de resíduos em nível municipal apresentado no PGIRS de São Paulo revela similaridade a um planejamento realizado sob os preceitos da AAE. A maioria dos elementos-chave preconizados como boas práticas de AAE encontra-se no PGIRS, à exceção de alguns pouco explorados ou ausentes. A interatividade entre atividades de avaliação e planejamento (conferência e comitês integrando diversos setores), mapeamento da situação ambiental (diagnóstico da geração de resíduos sólidos e identificação de problema de manejo e criação de cenários futuros), estratégias, metas, prazos, custos e competências (conjunto de estratégias para cada tipo de resíduo sólido urbano) são elementos-chave da AAE observados no PGIRS. Entre os aspectos pouco explorados encontram-se a participação pública, que, embora ampla, eximiu-se de um sistema de informações e divulgação sistemática, e a governança, que poderia ser ampliada para além dos atores envolvidos e para abarcar princípios, como o da transparência. Os elementos-chave de AAE ausentes estão associados à falta de delimitação objetiva dos fatores críticos para a decisão, à ausência de um quadro decisório estratégico para além das referências orientadas pelo próprio setor de resíduos e à carência de um sistema de indicadores ambientais para avaliação do plano e revisões futuras. Cabe destacar que o PGIRS apresenta diretrizes para a constituição de um sistema de informações sobre a gestão de resíduos sólidos, o SIMIRS-SP, com premissas que atendem à expectativa da AAE. Esse sistema de informações também seria útil para constituir a etapa de acompanhamento do PGIRS, não avaliada nesta pesquisa pela limitação do escopo, mas prevista e essencial para o sucesso da AAE.

Conclui-se que, mesmo sem diretrizes obrigatórias e formais para realizar a AAE no país, algumas das premissas mínimas essenciais do instrumento, como diagnóstico da situação, interatividade, opções estratégicas, cenários futuros e diretrizes para acompanhamento do plano, estão integradas ao PGIRS de São Paulo.

A estruturação do planejamento de resíduos sólidos no Brasil, levando-se em consideração as orientações preconizadas desde a PNRS até o PGIRS em nível municipal, permite constatar que o enfoque dado à hierarquia na gestão de resíduos - uma das principais estratégias da PNRS - demonstra que há nítida influência do nível decisório superior para o subsequente. Conclui-se que os conceitos de tiering estão presentes na cadeia decisória do planejamento e na gestão de resíduos sólidos, no caso do plano paulistano.

O PGIRS de São Paulo está orientado às boas práticas de AAE, enquanto elementos-chave esperados na aplicação do instrumento, e demonstra estar em consonância com os objetivos estratégicos da PNRS do planejamento de resíduos sólidos da escala nacional para a local, denotando tiering, também previsto como boa prática para o sucesso da AAE.

A AAE deve subsidiar o processo de planejamento, a execução e a avaliação sistemática de um plano. No PGIRS de São Paulo, considera-se que a aplicação formal da AAE pode contribuir para aprofundar aspectos não abordados e/ou não adequadamente abordados, ampliar o escopo dos aspectos abordados e internalizar benefícios comumente associados ao uso sistemático da AAE no processo de elaboração de planos municipais 
de gestão de resíduos sólidos. Como possíveis benefícios, destacam-se: a garantia de decisões transparentes e estratégicas, o aumento da participação pública, o fortalecimento do quadro de governança, a facilitação da integração vertical, horizontal e até diagonal no planejamento, entre vários outros.

Não obstante as semelhanças observadas entre PGIRS e AAE, o primeiro é um plano, e o segundo, um instrumento que subsidia a construção de planos que incluem adequadamente a temática ambiental e de sustentabilidade. Recomenda-se que a AAE seja utilizada no planejamento e na gestão de resíduos municipal nas revisões posteriores do PGIRS e na confecção desses planos municipais, por causa da capacidade limitada em planejamento da maioria dos municípios brasileiros. A utilização da AAE poderá ainda contribuir com etapas de realização do plano que fortaleçam a eficácia do planejamento e do tiering, não avaliada nesta análise. Recomenda-se, para as próximas revisões do PGIRS, a inclusão de indicadores de sustentabilidade voltados ao monitoramento e ao controle das ações propostas, além da criação de um sistema de informações sobre resíduos sólidos para a sociedade, incentivando a responsabilidade compartilhada pela gestão dos resíduos sólidos da cidade e a conscientização ambiental, tópicos previstos no plano, porém ainda não implantados.

\section{Agradecimentos}

A segunda autora agradece à Fundação de Amparo à Pesquisa do Estado de São Paulo (Fapesp), pelo apoio concedido ao financiamento da pesquisa que deu origem a este artigo científico, por meio do Processo Fapesp no 2015/20228-1, e ao Conselho Nacional de Desenvolvimento Cientifico e Tecnológico (CNPq), sob Processo no 309358/2017-5, pelo apoio ao desenvolvimento da pesquisa realizada.

\section{Referências}

Arts, J., Tomlinson, P., \& Voogd, H. (2011). Planning in tiers? Tiering as a way of linking SEA and EIA. In B. Sadler, R. Aschemann, J. Dusik, T. B. Fischer, M. Partidário, \& R. Berheem (Eds.), Handbook of strategic environmental assessment (pp. 415-436). New York: Routledge.

Associação Brasileira de Empresas de Limpeza Pública e Resíduos Especiais - ABRELPE. (2017). Panorama dos resíduos sólidos no Brasil 2016: panorama dos resíduos sólidos no Brasil 2016 (1. ed.). São Paulo: ABRELPE.

Bardin, L. (2009). Análise de conteúdo (1. ed.). São Paulo: Edições 10.

Brasil. (2010, 2 de agosto). Lei no 12.305, de 2 de agosto de 2010. Institui a Política Nacional de Resíduos Sólidos; altera a Lei no 9.605, de 12 de fevereiro de 1998; e dá outras providências. Brasília: Diário Oficial da União. Recuperado em 30 de julho de 2018, de http://www.planalto.gov.br/ccivil_03/_ato20072010/2010/lei/112305.htm

Brasil. Ministério do Meio Ambiente - MMA. ICLEI-Brasil. (2012). Planos de gestão de resíduos sólidos: manual de orientação. Brasília.

Bulkeley, H., \& Gregson, N. (2009). Crossing the threshold: municipal waste policy and household waste generation. Environment \& Planning A, 41(4), 929-945. http://dx.doi.org/10.1068/a40261.

Campos, H. K. T. (2012). Renda e evolução da geração per capita de resíduos sólidos no Brasil. Engenharia Sanitaria e Ambiental, 17(2), 171-180. http://dx.doi.org/10.1590/S1413-41522012000200006.

Desmond, M. (2009). Identification and development of waste management alternatives for Strategic Environmental Assessment (SEA). Environmental Impact Assessment Review, 29(1), 51-59. http://dx.doi.org/10.1016/j.eiar.2008.05.003.

Donnelly, A., Jones, M., O’Mahony, T., \& Byrne, G. (2007). Selecting environmental indicator for use in strategic environmental assessment. Environmental Impact Assessment Review, 27(2), 161-175.

http://dx.doi.org/10.1016/j.eiar.2006.10.006. 
Espinosa, D. C. R., \& Silvas, F. P. C. (2014). Resíduos sólidos: abordagem e tratamento. In A. Philippi Jr., M. A. Romério, \& G. C. Bruna (Eds.), Curso de gestão ambiental (2. ed., pp. 195-255). Barueri: Manole.

European Commission - EC. (2011, 21 de julho). Directive 2001/42/EC of the European Parliament and of the Council on the Assessment of the Effects of Certain Plans and Programmes on the Environment. Luxembourg: Official Journal of the European Communities. Recuperado em 30 de julho de 2018, de http://ec.europa.eu/environment/archives/eia/pdf/030923_sea_guidance.pdf

Fischer, T. B., Potter, K., Donaldson, S., \& Scott, T. (2011). Municipal waste management strategies, strategic environmental assessment and the consideration of climate change in England. Journal of Environmental Assessment Policy and Management, 13(4), 541-565. http://dx.doi.org/10.1142/S1464333211004000.

Gallardo, A. L. C. F., Duarte, C. G., \& Dibo, A. P. A. (2016). Strategic environmental assessment for planning sugarcane expansion: a framework proposal. Ambiente \& Sociedade, 19(2), 67-92. http://dx.doi.org/10.1590/1809-4422ASOC127007V1922016.

Hoornweg, D., \& Bhada, P. (2012). What a waste: a global review of solid waste management. Urban Development Series Knowledge Papers, 281(19), 1-44.

Instituto Brasileiro de Geografia e Estatística - IBGE. (2018). Brasil em síntese: São Paulo. Recuperado em 30 de julho de 2018, de https://cidades.ibge.gov.br/brasil/sp/sao-paulo/panorama

Jacobi, P. R., \& Besen, G. R. (2011). Gestão de resíduos sólidos em São Paulo: desafios da sustentabilidade. Estudos Avançados, 25(71), 135-158. http://dx.doi.org/10.1590/S0103-40142011000100010.

Josimović, B., Maric, I., \& Milijic, S. (2015). Multi-criteria evaluation in strategic environmental assessment for waste management plan, a case study: the city of Belgrade. Waste Management, 36, 331-342.

http://dx.doi.org/10.1016/j.wasman.2014.11.003. PMid:25464940.

Maiello, A., Britto, A. L. N. P., \& Valle, T. F. (2018). Implementação da Política Nacional de Resíduos Sólidos. Revista de Administração Pública, 52(1), 24-51. http://dx.doi.org/10.1590/0034-7612155117.

Malvestio, A. C., Gomes, P. M., \& Peixoto, D. J. O. (2012). Avaliação Ambiental Estratégica aplicada ao planejamento de resíduos sólidos no Brasil. In Anais da 2ª Conferência da REDE de Língua Portuguesa de Avaliação de Impactos e 1 a Cogresso Brasileiro de Avaliação de Impacto (pp. 13). São Paulo: Associação Brasileira de Avaliação de Impacto.

Marchi, C. M. D. F. (2015). Novas perspectivas na gestão do saneamento: apresentação de um modelo de destinação final de resíduos sólidos urbanos. urbe. Revista Brasileira de Gestão Urbana, 7(1), 91-105.

http://dx.doi.org/10.1590/2175-3369.007.001.A006.

McGimpsey, P., \& Morgan, R. K. (2013). The application of strategic environmental assessment in a non-mandatory context: regional transport planning in New Zealand. Environmental Impact Assessment Review, 43, 56-64. http://dx.doi.org/10.1016/j.eiar.2013.05.007.

Montaño, M., Oppermann, P., Malvestio, A. C., \& Souza, M. P. (2014). Currente state of the SEA system in Brazil: a comparative study. Journal of Environmental Assessment Policy and Management, 16(02), 1450022. http://dx.doi.org/10.1142/S1464333214500227.

Monteiro, M. B., \& Partidário, M. R. (2017). Governance in Strategic Environmental Assessment: lessons from the Portuguese practice. Environmental Impact Assessment Review, 65, 125-138.

http://dx.doi.org/10.1016/j.eiar.2017.04.007.

Nadruz, V. N., Gallardo, A. L. C. F., Montaño, M., Ramos, H. R., \& Ruiz, M. S. (2018). Identifying the missing link between climate change policies and sectoral/regional planning supported by Strategic Environmental Assessment in emergent economies: lessons from Brazil. Renewable \& Sustainable Energy Reviews, 88, 46-53. http://dx.doi.org/10.1016/j.rser.2018.02.006.

Oliveira, T. B., \& Galvão, A. D. C., Jr. (2016). 0 planejamento municipal na gestão dos resíduos sólidos urbanos e na organização da coleta seletiva. Engenharia Sanitaria e Ambiental, 21(1), 55-64. http://dx.doi.org/10.1590/S141341520201600100155929.

Organização das Nações Unidas - ONU. (1992). Agenda 21. Rio de Janeiro: ONU. 
Partidário, M. R. (2012). Strategic Environmental Assessment better practice guide: methodological guidance for strategic thinking in SEA. Lisboa: Portuguese Environment Agency e Redes Energéticas Nacionais.

Partidario, M. R., \& Sheate, W. R. (2013). Knowledge brokerage: potential for increased capacities and shared power in impact assessment. Environmental Impact Assessment Review, 39, 26-36.

http://dx.doi.org/10.1016/j.eiar.2012.02.002.

Philippi, A., Jr., \& Aguiar, A. O. (2018). Política nacional, gestão e gerenciamento de resíduos sólidos. In A. Philippi Jr., (Ed.), Saúde, saneamento e meio ambiente (pp. 321-377). Barueri: Manole.

Rega, C., \& Baldizzone, G. (2015). Public participation in Strategic Environmental Assessment: a practitioners' perspective. Environmental Impact Assessment Review, 50, 105-115. http://dx.doi.org/10.1016/j.eiar.2014.09.007.

Rizzo, H. B., Gallardo, A. L. C. F., \& Moretto, E. M. (2017). Avaliação Ambiental Estratégica e o planejamento do setor de trasnportes paulista. Engenharia Sanitaria e Ambiental, 22(1)

Salhofer, S., Wassermann, G., \& Binner, E. (2007). Strategic environmental assessment as an approach to assess waste management systems: experiences from an Austrian case study. Environmental Modelling \& Software, 22(5), 610-618. http://dx.doi.org/10.1016/j.envsoft.2005.12.031.

Sánchez, L. E. (2017). Por que não avança a avaliação ambiental estratégica no Brasil? Estudos Avançados, 31(89), 167-183. http://dx.doi.org/10.1590/s0103-40142017.31890015.

Sánchez, L. E., \& Silva-Sánchez, S. S. (2008). Tiering strategic environmental assessment and project environmental impact assessment in highway planning in São Paulo, Brazil. Environmental Impact Assessment Review, 28(7), 515522. http://dx.doi.org/10.1016/j.eiar.2008.02.001.

São Paulo. Prefeitura Municipal. (2014). Plano de Gestão Integrada de Resíduos Sólidos da Cidade de São Paulo. São Paulo: Comitê Intersetorial para a Política Municipal de Resíduos Sólidos. Recuperado em 30 de julho de 2018, de http://www.prefeitura.sp.gov.br/cidade/secretarias/upload/servicos/arquivos/PGIRS-2014.pdf

Silva, C. L., Fugii, G. M., \& Santoyo, A. H. (2017). Proposta de um modelo de avaliação das ações do poder público municipal perante as políticas de gestão de resíduos sólidos urbanos no Brasil: um estudo aplicado ao município de Curitiba. urbe. Revista Brasileira de Gestão Urbana, 9(2), 276. http://dx.doi.org/10.1590/21753369.009.002.ao09.

Tang, Z. (2008). Integrating the principles of strategic environmental assessment into local comprehensive land use planning. Journal of Environmental Assessment Policy and Management, 10(2), 143-171. http://dx.doi.org/10.1142/S1464333208003044.

Tetlow, M., \& Hanusch, M. (2012). Strategic environmental assessment: the state of the art. Impact Assessment and Project Appraisal, 30(1), 15-24. http://dx.doi.org/10.1080/14615517.2012.666400.

Turco, L. E. G., \& Gallardo, A. L. C. F. (2018). Avaliação de Impacto Ambiental e Avaliação Ambiental Estratégica: há evidências de tiering no planejamento de transportes paulista? Gestão \& Regionalidade, 34(101), 40-55. http://dx.doi.org/10.13037/gr.vol34n101.3878.

United Nations Environmental Program - UNEP. 2011). Towards a green economy: pathways to sustainable development and poverty eradication: sustainable development. Nairóbi: UNEP.

White, L., \& Noble, B. F. (2013). Strategic environmental assessment for sustainability: a review of a decade of academic research. Environmental Impact Assessment Review, 42, 60-66.

http://dx.doi.org/10.1016/j.eiar.2012.10.003.

Zanta, V. M., \& Ferreira, C. F. A. (2003). Gerenciamento integrado de resíduos sólidos urbanos. In A. C. Borges (Ed.), Resíduos sólidos urbanos: aterro sustentável para municípios de pequeno porte (pp. 1-18). São Carlos: Rima Artes e Textos.

Editor: Paulo Nascimento Neto

Recebido: Mar. 19, 2019

Aprovado: Abr. 14, 2019 